\title{
Prognosis of Cancer - A Semi Markov Process
}

\author{
Manjula S Dalabanjan, Pratibha Agrawal, Deepthi T, M. D. Suranagi
}

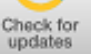

Abstract: Cancer begins in cells, the building blocks that make up tissues. Tissues make up the organs of the body. The buildup of extra cells often forms a mass of tissue called a growth, polyp or tumor. Tumors can be benign (non cancerous) or malignant (cancerous). Benign tumors are not as harmful as malignant tumors. The transformation of normal cells into cancer cells is called Carcinogenesis.Cancer is one of the major health problems persisting world-wide. Urbanization, industrialization, changes in lifestyles, population growth and ageing all have contributed for epidemiological transition in the country. The absolute number of new cancer cases is increasing rapidly due to growth in size of the population The stages of cancer are considered as different states of a Markov Process. Discrete-time Markov chains have been successfully used to investigate treatment programs and health care protocols for chronic diseases like HIV, AIDS, Hypertension etc. In this study, the process of carcinogenesis was classified into 6 states. The history of every patient is recorded in the form of a data segment starting from initial state.The transitional states and absorbing states are well defined. Since all the patients under study do not reach the last state at a given point of time, the process was studied as a Semi Markov Process. Maximum likelihood estimation of the transitional probabilities, the survival function, the hazard function and the waiting time distribution of patients in different states were studied. This kind of statistical methodology used to study the prognosis of cancer can be applied to real-time data of cancer patients.

Keywords: Markov Property, Cancer, Stages, Transition Probabilities, Maximum Likelihood Estimation, Semi Markov Model, Distribution function. AMS Classification: $62 G 32$

\section{INTRODUCTION}

Chronic diseases like cancer, HIV, etc. were often described in terms of different health stages as time progresses. Markov Chain is a simple and powerful model to describe progression through states. We study the progression of the disease by using Matrix Analysis. The key to Markov Model is the Markov Property. The property is that given the entire past history of a subject, the present state depends only on the recent past. This memory-less property allows the model to be described solely in terms of a transition matrix.

\section{REVIEW OF LITERATURE}

Definition: (Anderson, Goodman [1])

Manuscript received on May 26, 2021.

Revised Manuscript received on June 02, 2021.

Manuscript published on June 30, 2021.

* Correspondence Author

Manjula S Dalabanjan, Associate Professor, Department of Mathematics, DBIT, Bengaluru, India. Email: msd2670@gmail.com Bengaluru, India. Email:pratibhastatistics@gmail.com

Deepthi T*, Assistant Professor, Department of Mathematics, BIET, Hyderabad, India. Email: deepthitogercheti@gmail.com

M. D. Suranagi, Professor, Veterinary College, Karnataka Veterinary, Animal and Fisheries Sciences University, Bidar, India. Email: mdsuranagi@gmail.com

(C) The Authors. Published by Blue Eyes Intelligence Engineering and Sciences Publication (BEIESP). This is an open access article under the CC BY-NC-ND license (http://creativecommons.org/licenses/by-nc-nd/4.0/)
Pratibha Agrawal, Professor, Department of Mathematics, AMCEC,

$$
\begin{aligned}
& \text { Markov Chain: Consider a sequence of } \\
& \text { states } X_{1}, X_{2} \ldots X_{t} \\
& \begin{array}{l}
X_{t}=X_{t}, X_{t-1}=x_{t-1}, \ldots . X_{1}=x_{1} . \\
X=\left\{X_{1}, X_{2} \ldots\right\}_{\text {be a random process. }} \text { The random }
\end{array}
\end{aligned}
$$
process is called a Markov chain if it satisfies the Markov property.

$$
P\left\{X_{t+1}=x_{t+1} / X_{t}=x_{t}, \ldots . X_{1}=x_{1}\right\}=P\left\{X_{t+1}=x_{t+1} / X_{t}=x_{t}\right\}
$$

For every sequence $\left\{X_{1}, X_{2} \ldots . . X_{n}\right\}$ of elements of $\varepsilon$ and for every t, if the state space $\varepsilon$ is finite, let us write it as $\varepsilon=\{0,1, \ldots . . S\}$. Given a set of transition probabilities, it is often useful to collect these probabilities in a matrix.

After acute exposure to mutagens, Heidenrich et al. [2] derived expressions for the Armitage Doll hazard function.

The first one hit, two-stage mutation model of carcinogenesis is due to Armitage et al. [3]. This model is characterized by the deterministic assumption that the clone of first-order mutants grows exponentially.

Kendall, Haris [4] studied the prognosis of cancer as a birth and death process and deduced a formula for the hazard function.

Rafael Meza [5] studied the history of multistage carcinogenesis models in his article "Stochastic modeling of Carcinogenesis". He studied the "Two-Stage Clonal Expansion Model" given by Moolgavkar, Venzon [6] considering once as a non-homogeneous Poisson Process and another time as a continuous-time Markov process.

Heidenreich et al. [7] analyzed population-level data and derived the closed-form expressions for the hazard and survival functions in the case of constant and piecewise constant parameters. Also analyzed the experimental data and obtained numerical solutions in the case of general agedependent parameters.

Alwell et al. [8] studied the spread of infectious disease in a community. The progression of infectious disease was modeled using a technique of branching process with immigration.

Jerzy, Elizabeth [9] carried out an experimental study with the tumors in mice. The tumors were found on the surface or within the lungs of mice. Jerzy, Elizabeth [9] considered only those tumors which contained $n$ cells and denoted $\pi \mathrm{n}$ as the probability that a tumor is composed of exactly $\mathrm{n}$ cells. As $\mathrm{n}$ grows, $\pi \mathrm{n}$ never decreases and eventually tends to unity. Broadly speaking, they were attempting to construct a model of carcinogenesis representing a combination of several births and death stochastic processes such that the consequences of the model agree, at least qualitatively, with certain empirical findings.

\section{Published By:}

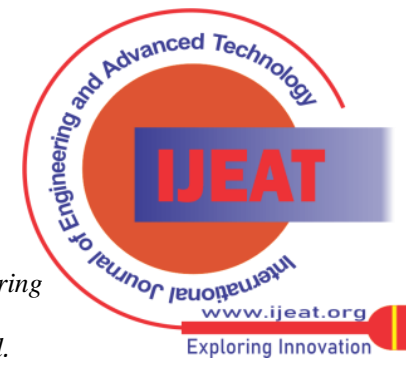


Rafael, Joanne [10] analyzed the data from 19732010. In the SEER-9 registry, the incidence rates of thyroid cancer are available. Using the multistage models, they studied how the i) malignant conversion rates of thyroid tumors were influenced by sex, race and histology, ii) promotion of thyroid cancer is also influenced by sex, race and history.

Lagakos et al. [11] studied a stochastic model and its use in the analysis of censored multistate data. The model proposed by Lagakos et al. [11] is based on the semiMarkov process in which state changes from an embedded Markov Chain and where waiting time between two jumps depends only on the adjoining states, also considered that a subject begins in one of several states and that at each point of time will be in one of the "s" possible states, denoted as $1,2,3, \ldots \mathrm{s}$. The states $1,2, \ldots \mathrm{s} 1$ were considered to be as transient states and the states $s 1+1, \ldots . s$ to be absorbing states. Author determined the general likelihood function for a set of partially censored observations and Maximum Likelihood Estimates (MLE) of the model were found.

The covariance matrix of the probability vectors of the Markov process was derived by Dinse and Larson [12]. They also focussed on the tests of equality of the 'conditional sojourn time distributions. These comparisons were useful for them to carry out inferences about the timedependent nature of events defining the multistate stochastic process.

An alternative approach utilizing cause-specific hazard functions for observable quantities, including timedependent covariates was proposed by Prentice et al. [13]. A method involving the estimation of parameters that relate time-dependent risk indicators for some causes to causespecific hazard functions was proposed for the study of interrelations among failure types.

\section{METHODOLOGY}

In the present study, we modify the work proposed by Bruce, Peter [14] by including an initial probability vector. With the motivation of work done by Sudhendu [15], the cancer disease is classified into 6 states. Suppose each patient begins in one of the six states and that at each point of time, will be in one of the possible six states denoted by $S_{1}, S_{2}, S_{3}, S_{4}, S_{5}, S_{6}$, the prognosis of cancer disease as a Markov chain is studied under the following assumptions:

a) States 1 to 5 are transitional states, where $S_{1}$ to $S_{4}$ are four stages of cancer, $S_{5}$ the state, "cured" and $S_{6}$ the state, "death" of a person.

b) $S_{6}$ is an absorbing state. Once the patient enters the state $S_{6}$ he remains in the same state.

c) Distributions of the duration of time in each of the states are independent.

d) There is no transition from states $S_{5}$ to $S_{1}, S_{5}$ to $S_{4}, S_{4}$ to $S_{3}, S_{3}$ to $S_{2}$ and $S_{2 \text { to }} S_{1}$.

\section{SEMI MARKOV MODEL}

Let $X_{t}$ denote the cancer stage (state) of a patient at time $t$. The change of state of cancer from one state to another or to the state $S_{6}$ is considered as an event. We aim to find the distribution function of duration of stay of a patient in different states. The different states of cancer, $S_{1}, S_{2} S_{3}, S_{4}$, S5are transient statesand S6 is an absorbing state as defined in the Model A. The state space

$\mathrm{S}=\{\mathrm{S} 1, \mathrm{~S} 2, \mathrm{~S} 3, \mathrm{~S} 4, \mathrm{~S} 5, \mathrm{~S} 6\}$.

The work is in line with Dinse and Larson [12] and Lagakos et al. [11]. Let Fij(t) denote the distribution function of duration of stay in state Si before going to state $\mathrm{Sj}$. The variable is $\mathrm{Xt}$ considered only at jump points, giving rise to $\mathrm{X} 1, \mathrm{X} 2, \mathrm{X} 3 \ldots$

The system starts in a state $\mathrm{X} 0$, stays there for a length of time, moves to another state, stays there for a length of time, etc. This system or process is called a semi- Markov process.

When Fij(t) is anarbitrary continuous distribution, the process is Semi-Markov. In model C, let us assume Xt is homogeneous.

Let Tkrepresent waiting time between (k-1)th and kth state at which $\mathrm{Xt}$ jumps from Xk-1to $\mathrm{Xk}$. The Tk's are assumed to be independent. Thus the transition probabilities of the process is given by

$$
P_{i j}=\operatorname{Pr}\left\{X_{k+1}=j \mid X_{k}=i\right\} \text {, }
$$

And the waiting time distributions are determined by

$$
\overline{F_{i j}}(t)=\operatorname{Pr}\left\{T_{k+1}>t \mid X_{k}=i, X_{k+1}=j\right\}
$$

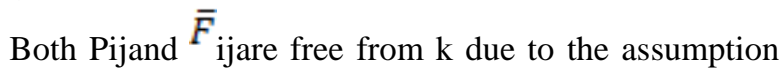
that Xt are homogeneous. We consider Xt as Semi- Markov process which can be studied at the states $\mathrm{S} 1, \mathrm{~S} 2, \mathrm{~S} 3, \ldots$

\subsection{History of the Data}

The Prognosis of cancer starts from Stage $1\left(S_{1}\right)$ and ends in Stage $6{ }^{\left(S_{6}\right)}$. There may be some patients who have not reached the state $S_{6}$ at the time of our data analysis, so the data is right censored partially.

Suppose there are $N$ patients in the study, at a given point of time $\tau$, and that history of ith patient is written using the data segment

$$
\begin{aligned}
& \qquad \mathrm{Di}=\{\mathrm{X} 0, \mathrm{~T} 1, \mathrm{X} 1, \mathrm{~T} 2, \mathrm{X} 2, \mathrm{~T} 3, \ldots \ldots \ldots \ldots \\
& \ldots \mathrm{Xm}-1, \mathrm{Tm}, \mathrm{Xm}\} \\
& \text { with } 1 \leq X_{j} \leq S_{5}, 0 \leq j \leq m-1 \\
& \text { Here } X_{m}=S_{6} \text { if the ith Patient has expired by }
\end{aligned}
$$
time $\tau$. The time instant $\tau$ is considered to be the point of time to carry out data analysis. Since S6is an absorbing state a data set $D_{i}$ is complete for ithpatient if $X_{m}=S_{6}$. However some patients may not have reached the state S6at the time $\tau$, such patients may be present in any of the transient states S1, S2, S3, S4, S5 at the time $\tau$, then we write

\section{Published By:}

Blue Eyes Intelligence Engineering and Sciences Publication

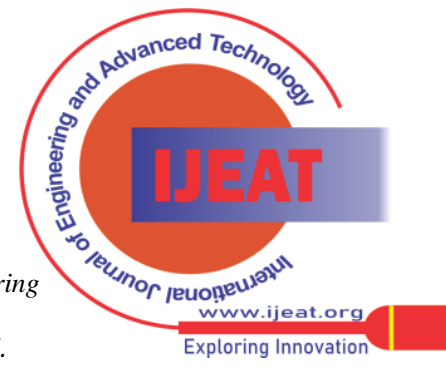




$$
\begin{aligned}
& X_{m}=\left\{\begin{array}{llll}
S_{6} & \text { if } & D_{i} & \text { is complete } \\
S_{6}+1 & \text { if } \quad D_{i} & \text { is right censored }
\end{array}\right. \\
& \mathrm{i}=1,2,3 \ldots \ldots \mathrm{N}
\end{aligned}
$$

Reporting time of a patient to the doctor will be usually once in a month. The set of waiting times $\{\mathrm{Tk}\}$ in $\mathrm{D}$ may be classified through an appropriate frequency distribution. Let $\mathrm{u} 1<\mathrm{u} 2<{ }_{\mathrm{u} 3 \ldots \ldots<\mathrm{uc} \text { denote } \mathrm{c} \text { distinct ordered (increasing) }}$ waiting times observed across all the patients under study. Let mijhbe frequency of uh among transitions from State Si to State $S \mathrm{j}$ of length uh.

$$
\mathrm{i}=1,2,3,4,5 ; \mathrm{j}=1,2,3,4,5,6 ; \mathrm{h}=1,2,3,4 \ldots . \mathrm{c} \text {. }
$$

Themijh gives the number of waiting times from $\mathrm{Si}$ to $\mathrm{Sj}$ of duration uh in the data set $\mathrm{D}$ on $\mathrm{N}$ Patients. For the censored data segments in $\mathrm{D}$, let $\mathrm{mi}$, S6+1,hdenote the number of waiting times in state Sithat are censored in [uh, $\mathrm{uh}+1), \mathrm{h}=0,1,2, \ldots . \mathrm{c}$ with $\mathrm{u} 0=0$ and $\mathrm{uM}+1=\infty$.

\subsection{Parameterization} $\overline{F_{i j}}($.

The process Xt which is determined by $\mathrm{P}(\mathrm{i}), \mathrm{P}(\mathrm{i}, \mathrm{j})$, and can be parameterized through characterisation in terms of a set of hazard functions $\theta_{i j h}$ which are functions of transition probabilities $P_{i j}$ and waiting time distributions Fij. Following such characterization, the Maximum Likelihood Estimates of $\theta_{i j h}$ and related survival functions can be derived. This approach follows Dinse and Larson [12].

Consider waiting times $\{\mathrm{Tk}\}$ as discrete random variable with distribution at the observed waiting times u1, u2,...uc of $\mathrm{N}$ patients. Prentice et al. [13], defined the Discrete Event-Specific Hazard Function by

$$
\begin{gathered}
\begin{array}{c}
\theta_{i j h}=\operatorname{Pr}\left\{T_{k+1}=u_{h}, X_{k+1}=S_{j} \mid T_{k+1} \geq u_{h}, X_{k}=S_{i}\right\} \\
\quad \ldots .(4)
\end{array} \\
\quad \sum_{j=1}^{6} \theta_{i j h} \text { represent hazard function at uh, when the }
\end{gathered}
$$
person at state Simove to

$\mathrm{Si}+1, \ldots$ or to $\mathrm{S} 6$.

Let us define the survival function for waiting times in state i by

$$
S(t ; i)=\operatorname{Pr}\left\{T_{k+1}>t \mid X_{k}=S_{i}\right\}_{\mathrm{i}=1,} 2, \quad 3,4, \quad 5,6
$$

$\mathrm{S}(\mathrm{t}$; i) gives the probability that a patient in state Sicontinues to be in the same state after time t.

Since we have defined $\mathrm{Tk}$ as discrete distribution of time taking the values $\mathrm{u} 1, \mathrm{u} 2$.....uh , $\mathrm{S}(\mathrm{t}$; i) becomes a step function. Thus,

$$
S\left(u_{h} ; i\right)=\operatorname{Pr}\left\{T_{k+1}>u_{h} \mid X_{k}=S_{i}\right\}
$$

The survival function $\mathrm{S}(\mathrm{t} ; \mathrm{i})$ for waiting times in state i can be expressed as

$$
S(t ; i)=\frac{S\left(u_{1} ; i\right)}{S\left(u_{0} ; i\right)} \frac{S\left(u_{2} ; i\right)}{S\left(u_{1} ; i\right)} \ldots \ldots \ldots \ldots . . . \frac{S\left(u_{h} ; i\right)}{S\left(u_{h-1} ; i\right)}
$$

$$
\text { For } 0 \leq u_{0} \leq u_{1} \leq \ldots \ldots . . u_{h-1} \leq u_{h}
$$

Let qih denote the conditional probability that waiting time in state $S_{i}$ exceeds $u_{h}$, given that it exceeds uh-1. Therefore using a definition of $\mathrm{S}(\mathrm{uh} ; \mathrm{i})$ and $\theta_{i j h}$ we get

$$
q_{i h}=\frac{S\left(u_{h} ; i\right)}{S\left(u_{h-1} ; i\right)}
$$

$$
\left(\theta_{i 1 h}+\theta_{i 2 h}+\ldots \ldots \ldots \theta_{i j h}\right)
$$

$$
\sum_{j=1}^{6} \theta_{i j h}
$$$$
=1-
$$

From equation (6),

$$
S(t ; i)=\prod_{r=1}^{h} q_{i r}
$$

With $\mathrm{uh} \leq \mathrm{t} \leq \mathrm{uh}-1$, for some $\mathrm{h}=0,1,2, \ldots$.M

\section{THE LIKELIHOOD FUNCTION}

Let $D^{c}$ and $D^{r c}$ be segment of data set Dicorresponding to completed and right censored ones such that

$$
D=D^{c} \cup D^{r c}
$$

Since $D^{c} D^{r c}$ are independent corresponding to $N$ different patients the likelihood function of $\theta_{i j h}$ given the data set $\mathrm{D}$ can be factored as

$$
{ }_{L}\left\{\theta_{i j h} \mid D\right\}=L\left\{\theta_{i j h} \mid D^{c}\right\} L\left\{\theta_{i j h} \mid D^{r c}\right\}
$$

Corresponding to completed and right-censored histories,

$$
\begin{aligned}
& \left\{\theta_{i j h} \mid D^{c}\right\} \alpha \prod_{h=1}^{M} \prod_{i=1}^{6} \prod_{j=1}^{6}\left(\theta_{i j h}\right)^{m_{i j h}} \\
& m_{66 h}=0 \text { for all h. } \\
& \text { with } \\
& \text { To obtain the second factor consider the } \\
& \sum_{r=h}^{M} \sum_{j=1}^{6} m_{i j r} \text { is the total number of completed waiting }
\end{aligned}
$$

is the total number of completed waiting times greater than uhfollowing the disease is the state $\mathrm{Si}$ for the first time. This is the total number of patients who have expired by time $\tau$, who move from state Sjafter staying in the state Si for uh or more months. It is obtained from Dc.

$$
\text { The sum } \sum_{r=h}^{M} \sum_{j=1}^{6} m_{i, s_{6}+1, r}
$$

is the total number of rightcensored time $\left(\geq u_{h}\right)$ spent in state Si.

\section{Published By:}

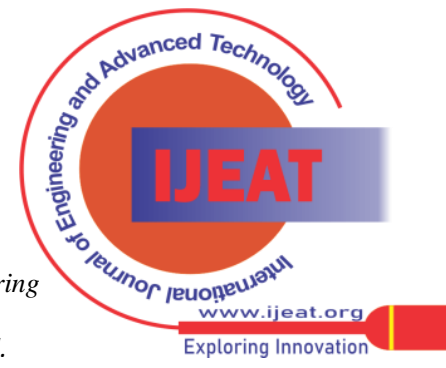


This is also the number of patients who at time $\tau$ are at state Si for uh or more months. It is obtained from Drc. Next, we define

$$
n_{i h}=\sum_{r=h}^{M} \sum_{j=1}^{6} m_{i j r}+\sum_{r=h}^{M} \sum_{j=1}^{6} m_{i, s_{6}+1, r}
$$

Then $n_{i h}$ gives the total numbers of patients in state $S_{i}$ who are possible to reach state $S_{6}$ after a stay of uh months in state $S_{i}$ the uncensored waiting time in state $S_{i}$ of length uh add to $\sum_{j=1}^{6} m_{i j h}$

And the Likelihood function corresponding to the censored part of the data

$$
L\left\{\theta_{i j h} \mid D^{r c}\right\} \alpha \prod_{h=1}^{M} \prod_{i=1}^{6}\left(1-\sum_{j=1}^{6} \theta_{i j h}\right)^{n_{i h}-\sum_{j=1}^{6} m_{i j h}}
$$

Combining (8) and (9) the log likelihood function for maximisation is

$$
l\left\{\theta_{i j h} \mid D\right\}=\sum_{h=1}^{M} \sum_{i=1}^{6}\left\{\left(n_{i h}-\sum_{j=1}^{6} m_{i j h}\right) \log \left(1-\sum_{j=1}^{6} \theta_{i j h}\right)+\sum_{j=1}^{6} m_{i j h} \log \left(\theta_{i j h}\right)\right\}
$$

\section{ESTIMATION OF PARAMETERS}

It is evident from expression (10) that maximisation of $l\left[\left(\theta_{i j h}\right) \mid D\right]_{\text {with respect }}\left(\theta_{i j h}\right)$ leads to Maximum Likelihood Estimate (MLE)

$$
\theta_{i j h}^{\Lambda}=\frac{m_{i j h}}{n_{i h}}
$$
$\hat{\theta}_{i j h}$ in expression for qih will be given
Substituting
in equation (7) as an MLE of qih will be

$$
\hat{q}_{i h}=1-\sum_{j=1}^{6} \hat{\theta}_{i j h}^{\Lambda}
$$

$\Lambda$

Similarly, substituting $\mathcal{q}_{i h}$ in the expression for $\mathrm{S}(\mathrm{t}$; i) the MLE of $\mathrm{S}(\mathrm{t} ; \mathrm{i})$ is

$$
\begin{gathered}
\Lambda \\
S(t ; i)=\stackrel{q}{i h}_{i h} q_{i h} \ldots \ldots \ldots . . q_{i h}, \\
u_{h} \leq X_{t} \leq u_{h-1}, \quad \text { for } \mathrm{h}=0,1,2, \ldots \ldots \mathrm{c}
\end{gathered}
$$

If the largest time in state $S_{i}$ is uncensored and otherwise also will be same asabove but reduces to zero for $t$ > UM . Since ${ }^{\Lambda}{ }_{i j h}$ is the proportion of patients who spent a duration of length uh in state Sjout of the patients who were in state $\mathrm{Si}, P_{i j h}$ is the probability that the patient already spent uh months in state Si before shifting to state $\mathrm{Sj}$. Therefore

$$
P_{i j h}=\operatorname{Pr}\left\{T_{k+1}=u_{h}, X_{k+1}=j \mid X_{k}=S_{i}\right\}
$$
1 ;) as

We can express the same in terms of $\theta_{i j h}$ and S(uh-

$$
P_{i j h}=\theta_{i j h} S\left(u_{h} ; i\right)
$$

The estimate of $P_{i j h}$ is obtained by substituting the MLE of $\theta_{i j h}$ and S(uh-1; i) in equation (11)

$$
\stackrel{\Lambda}{P}_{i j h}=\stackrel{\Lambda}{\theta}_{i j h}{ }_{S}^{S}\left(u_{h} ; i\right)
$$

Expressing $\mathrm{P}(\mathrm{i}, \mathrm{j})$ in terms of Pijhwe obtain the MLE of $P(i, j)$ as

$$
\hat{\boldsymbol{P}}(i, j)=\sum_{h=1}^{M} \hat{P}_{i j h}^{\Lambda}
$$

Similarly, the MLE of Fij (t) is obtained as

$$
\hat{F}_{i j}(t)=\left\{\hat{P}(i, j)-\sum_{h: u_{h} \leq t} \hat{P}_{i j h}\right\} / \hat{P}(i, j)
$$

If we have longitudinal data of a cohort of $\mathrm{N}$ patients who visit the doctor every month, the transition probabilities of stages of cancer and the distribution of $\{\mathrm{Tk}\}$ can be estimated for a real-life problem.

\section{CONCLUSION}

In this article, Semi-Markov Process is proposed to study the process of carcinogenesis.

- If we have longitudinal data where patients are followed through their transitions from one state to another, then we can find the transitional probabilities to go from one state to another. Alwell et al. [8] used the data from Oct/Nov 1967 epidemic of respiratory disease in Tristanda Cunha which contained the number of persons suffering from infectious disease and number of persons susceptible to infections throughout 16-time points and forecasted the future spread of the disease using a proposed forecast function.

- For an illustration of our study, one can consider a clinical trial of cohort of patients, corresponding to different states $S_{1}, S_{2}, S_{3}, S_{4}, S_{5}, S_{6}$ then in due course of time, the prognosis of disease can be studied for every fixed interval of time. The probability of their transition to the next possible state can be found using this data. We can also find the distribution of waiting time of patients $\left\{T_{k}\right\}$ in the states $S_{i}, i=1,2$, $3,4,5$.

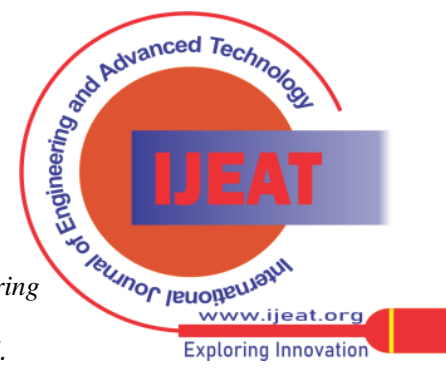




\section{REFERENCES}

1. Anderson T W , Goodman L A, Statistical Inference about Markov Chains. Ann Math Stat 1957; 28: 89-110

2. W. F. Heidenreich, E. G. Luebeck, and S. H. Moolgavkar, Some properties of the hazard junction of the two-mutation clonal expansion model, Risk Anal., Vol. 17, (1997), pp. 391-399.

3. Armitage P. and R. Doll, A two stage theory of carcinogenesis in relation to the age distribution of human cancer, British Journal of cancer, Vol.11, (1957), pp. 161-169.

4. D. G. Kendall, Birth and death processes, and the theory of carcinogenesis, Biometrika, Vol. 47, (1960), pp. 13-21.

5. Rafael Meza, Stochastic modeling of Carcinogenesis, BMC Public Health (2010).

6. Moolgavkar SH \& Venzon DJ. Two-event models for carcinogenesis: incidence curves for childhood and adult tumors. Mathematical BiosciencesVol. 45, (1979), pp.55-77.

7. W. F Heidenreich, P. Jacob, D. G. Kendall, Birth-and-death processes, and the theory of carcinogenesis, Biometrika, Vol. 47 (1960), pp. 13-21

8. Alwell J. Oyet, Brajendra C. Sutradhar, Longitudinal modeling of infectious disease. Sankhya: The Indian Journal of Statistics, Vol. 75-B, Part 2, (2013) pp. 312-342.

9. Jerzy. Neyman and Elizebeth. Scott, Statistical aspects of the problem of carcinogenesis, in Proceedings of the Fifth Berkeley Symposium on Mathematical Statistics and probability. University of California Press, (1967), pp. 745-776

10. Rafael Meza and Joanne T. Chang Multistage Carcinogenesis and the incidence of thyroid cancer in the US by sex, stage and histology. BMC Public Health (2015).

11. Lagakos, Sommer, Jelen, Semi Markov Models for Partially Censored Data. Biometrika, 65, (1978), pp. 111-117.

12. Dinse and Larson, A Note on Semi Markov Models for Partially Censored Data, Biometrika, 73,(1986), pp. 379-386

13. Prentice R. L., The analysis of failure time in the presence of competing risks, Biometrics 34, (1978), pp. 541-554.

14. Bruce A. Craig and Peter P. Sendhi, Estimation of the transition matrix of a discrete- time Markov Chain, Health Economics, Vol. 11 (2002).

15. Sudhendu Biswas, Applied of Stochastic process, A biological and population oriented approach New Age International Publications Ltd.(2013)

\section{AUTHORS PROFILE}

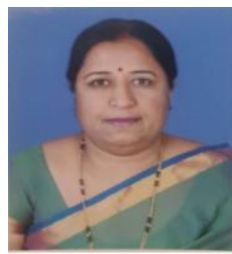

Dr. Manjula S Dalabanjan, is working as Associate Professor in the Department of Mathematics, Don Bosco Institute of Technology Bengaluru. She has over 25 years of teaching experience and 10 years of research experience. She has published 4 papers in International Journals and presented 9 papers different Conferences. She received her UG degree, PG Degree and PGDCA from Karnatak University Dharwad and Ph. D from VTU, Belagavi. The author is a life member of ISTE. Email: msd2670@gmail.com

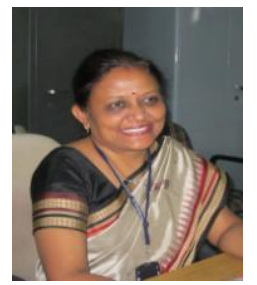

Dr. Pratibha Agrawal, is retired Professor from AMC Engineering College, Bengaluru. She has an experience of over 29 years of teaching in different institutions and 4 years of Industry. The author has published 8 papers in various journals and presented 9 papers in different conferences. Email: pratibhastatistics@gmail.com.

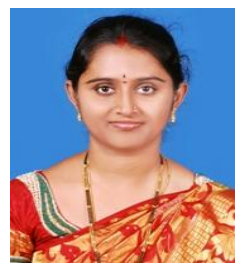

Dr. T. Deepthi, is working as Assistant Professor in the Department of Mathematics, Bharat Institute of Engineering and Technology, Hyderabad. She awarded Ph.D in Statistics in the year 2018 from University College of Science, Osmania University, Hydearbad. She has over 12 years of teaching experience and 4 years of research experience. She has published 6 papers in International Journals and presented 7 papers in International and National Conferences. During her tenure she had handled various statistics \& Mathematics papers for UG and PG Students and also for Pharmacy. The author is a life member for Society for Development of Statistics (SDS) and AADSOU. Email: deepthitogercheti@gmail.com

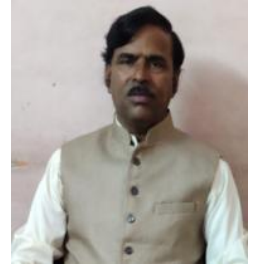

Dr. M.D. Suranagi, is working as Professor of Statistics, Veterinary College, Karnataka Veterinary, Animal and Fisheries Sciences University, Bidar involved in teaching of Statistics courses for the students of undergraduate and post graduate and also assisting in statistical analysis for the staff and students of the University since 1995. The Author has completed Five University projects. The Author has published more than 35 research papers in National and International Journals. The Author has visited Palestine during 2006 to attend the "Firstinternational conference on Mathematical Sciences" at Al-Azar University, Gaza, Palestine and presented a research paper in the International Conference. The Author is life Member of the Indian Society of Agricultural Statistics.

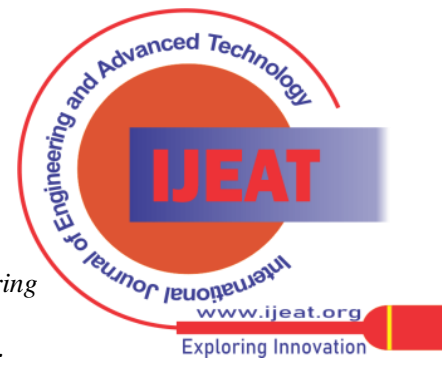

